Ann. Biol. anim. Bioch. Biophys., 1977, 17 (2), 153-163.

\title{
Etude de l'évolution de la nature des triglycérides du lait, pendant les trois premières semaines de lactation, chez la lapine normale ou tarie précocement
}

\author{
par J. P. PERRET, A. DORIER, C. BACQUES \\ avec la collaboration technique de Martine GILET \\ I. U. T. Biologie Appliquée, 43, bd du 11-Novembre-1จ18 \\ 69621 Villeurbanne
}

Summary. Milk triglyceride patterns during the first three weeks of lactation in the norma or early drying-off doe rabbit.

The triglyceride composition pattern of normal rabbit milk is compared with that of precocious drying-off ( 20 days of lactation) does to specify the modality of lipogenesis.

Normal milk is gradually enriched in short-chain fatty acids $(C \leqslant 10)$ as well as in short-chain triglycerides $(C \leqslant 46)$. But only the analysis of triglyceride groups can reveal significant differences between the first ten days of lactation and the following ten days.

During drying-off, milk exhibits most of the characteristics of end of lactation milk only after the first ten days. However, since early lactation, it shows a low $\mathrm{Ca}$ and shortchain triglyceride ratio which may be precursory signs of early drying-off.

Statistical analysis of the triglyceride composition of normal and pathological milk indicates that :

1) rabbit milk triglycerides have non-random fatty acid distribution or they are composed of several populations, each showing random fatty acid distribution ;

2) esterification process is constant during the overall period of lactation studied.

\section{I. - Introduction et objet du travail.}

L'analyse immédiate du laił de la lapine a été réalisée par de nombreux auteurs. Certains d'entre eux ont suivi, au cours d'une lactation normale, l'évolution des concentrations des principaux éléments : protéines, lactose : Cowie (1969) et Lebas (1971) ; matières grasses : Hall (1971) et Lebas ; éléments minéraux : Lebas, Besancon, Abouyoub (1971), et vitamines : Coates, Gregory, Thompson (1964).

Les phases d'activation de la lipidogenèse mammaire sont caractérisées pendant la gestation ef dès le début de la lactation par une hausse du taux des acides gras les plus courts (octa et décanoïque). Elles sont soumises à un contrôle hormonal établi, in vitro, par Carey (1973) et Dils (1972). Le mécanisme d'incorporation des acides gras 
dans les triglycérides du lait demeure inconnu. Smith, Watts et Dils (1968) ont seulement fourni des indications sur les proportions des divers groupes de triglycérides à partir de trois prélèvements $\in \mathrm{ff} \in$ ctués sur trois lapines.

La connaissance de l'évolution de la composition des triglycérides qui sont un des constituants maje urs du lait ( $\epsilon$ nviron 12 p. 100 du poids frais) et fourniss $\triangleq n t$ au lapereau l'essentiel de l'apport calorique alimentaire, est indispensable si on veut étudier la physio'ogie de la digestion du lait maternel et envisager de remplacer ce dernier par un laił synthétique.

Le lapereau peut être sevré à trois semaines, au cours de l'allaitement mixte. La glande mammaire de la lopine involue d'ailleurs rapidement au-delà du $20^{\mathrm{e}}-25^{\mathrm{e}}$ jour de lactation (Denamur, 1961). C'est la raison pour laquelle ce travail est destiné à l'étude des variations de la composition des triglycérides du lait au cours de la phase de lactogenèse ascendante.

Etant donné l'importance, mise en évidence dans le lait de vache, des équilibres entre les éléments minéraux, le lactose ef les protéines, nous avons mesuré simultanément l'évolution du taux de ces éléments.

Cette étude a été aussi effectuée chez des lapines atteintes de tarissement précoce, et nous a fourni des points de comparaison pouvant aider à la compréhension des modalités de la lipidogenèse mammaire.

\section{II. - Matériel et méthodes.}

\section{A. - Animaux.}

Nous avons utilisé 6 lapines primipares de race commune (Blanc de Bouscat) maintenues à l'intérieur d'un local climatisé à $20^{\circ} \mathrm{C}$ dans des cages de $1 \times 0,65 \mathrm{~m}$ séparées en deux parties égales par une grille.

La litière est composée de paille et les lapines reçoivent eau et granulés industriels ad libitum.

Mère et petits sont réunis $10 \mathrm{mn}$ chaque matin pour la tétée, après les prélèvements de lait sur la mère.

Le nombre de petits est limité à 8 pour chaque portée.

\section{B. - Prélèvements.}

Les échantillons de lait sont récoltés mécaniquement à l'aide d'une machine à traire simple proche de celle décrite par Appel (1971), et composée d'une ventouse en verre prolongeant une corne à vide adaptée sur un ballon à col rodé (CS 19/26) de $25 \mathrm{ml}$. La dépression est assurée par une trompe à eau et modulée manuellement par une prise d'air placée en dérivation sur le tube de caoutchouc reliant la trompe à eau à la trayeuse.

Pendant la traite, la lapine est maintenue « assise » par un opérateur ef la glande mammaire est massée modérément. La traite est stoppée dès l'obtention de $10 \mathrm{ml}$ de lait. Lapine et lapereaux sont alors réunis pour la tétée.

Trois de ces lapines $(=$ lot 1 ) ont mené toute leur portée à terme. Les petifs des trois autres $\left(=\right.$ lot 2 ) sont tous morts, entre le $8^{\mathrm{e}}$ et le $20^{\mathrm{e}}$ jour de la lactation, de 
malnutrition comme l'indiquent une croissance médiocre et la vacuité gastrique à l'autopsie. Nous n'avons pas caractérisé l'étiologie de ce tarissement précoce des lapines qui ne présentent aucun signe de pathologie quelconque et ont, par ailleurs, mené jusqu'au sevrage leur portée suivante.

Les mères ont ainsi été traites pendant trois semaines. Deux chaque jour. Les quatre autres tous les 3 ou 4 jours. Nos analyses portent sur 63 prélèvements.

$$
\text { C. - Dosages. }
$$

1) Les lipides sont extraits par la méthode de Delsal (1944), et les triglycérides analysés par chromatographie en phase gazeuse sur colonne de $50 \mathrm{~cm}$ de Gas-Chrom Q 100-120 mesh supportant 10 p. 100 en poids de S.E. 30. Le débit du gaz vecteur $\left(\mathrm{N}_{2}\right)$ est de $40 \mathrm{ml} / \mathrm{mn}$ à $230^{\circ} \mathrm{C}$, la température d'analyse est programmée de $230^{\circ} \mathrm{C}$ à $360^{\circ} \mathrm{C}$ à raison de $2,5 \circ \mathrm{C} / \mathrm{mn}$. Les coefficients de corrections pondéraux sont calculés après analyse d'un mélange-étalon de triglycérides saturés (Applied Sciences Laboratories $n^{\circ} \mathrm{A}$ et $C$ ), à partir des résultats fourris par un intégrateur digital.

Les acides gras constitutifs des triglycérides sont analysés après leur transformation en esters-méthyliques, à $180^{\circ} \mathrm{C}$, sur colonne de $3 \mathrm{~m}$ de Chromosorbe $\mathrm{W} 80$ 100 mesh supportant 20 p. 100 en poids d'E.G.S. On calcule de la même façon leurs coefficients de correction pondéraux.

2) Les principaux éléments minéraux $(\mathrm{Na}, \mathrm{K}, \mathrm{Ca})$ sont dosés par photométrie de flamme après défécation trichloracétique [méthode de Guéguen et Rombauts (1961)].

3) Le lactose est dosé selon la méthode de Dorche (1951). Les résultats expriment la teneur en lactose anhydre.

4) On détermine la teneur en protéines totales puis en caséine après précipitation par l'acide acétique à $\mathrm{pH} \mathrm{4,6}(\mathrm{N}$ Kjeldahl $\times$ 6,38).

D. - Analyse des résultats.

1) L'analyse statistique des résultats a été effectuée au moyen du test « $\boldsymbol{t}$ » de Student appliqué aux petits échantillons après comparaison des variances. Sont considérées comme significativement différentes, les moyennes pour lesquelles : $\alpha<0,01$.

2) Pour déterminer les proportions pondérales ou molaires théoriques calculées des groupes de triglycérides, si on suppose une répartition au hasard de leurs acides gras constifutifs, on utilise le mode de calcul décrit par Kuksis, Mc Carthy et Beveridge (1963).

3) On définit l'indice de spécificité global des triglycérides selon Watts, Dils et Wehr (1972), par la somme des différences négatives ou positives entre le pourcentage molaire calculé et le pourcentage molaire observé pour chaque groupe de triglycérides.

\section{III. - Résultats.}

A. - 1) Les teneurs en protéines, lactose, matières grasses, sodium ne varient pas de façon significative pendant la phase de lactogenèse ascendante (lot 1). 


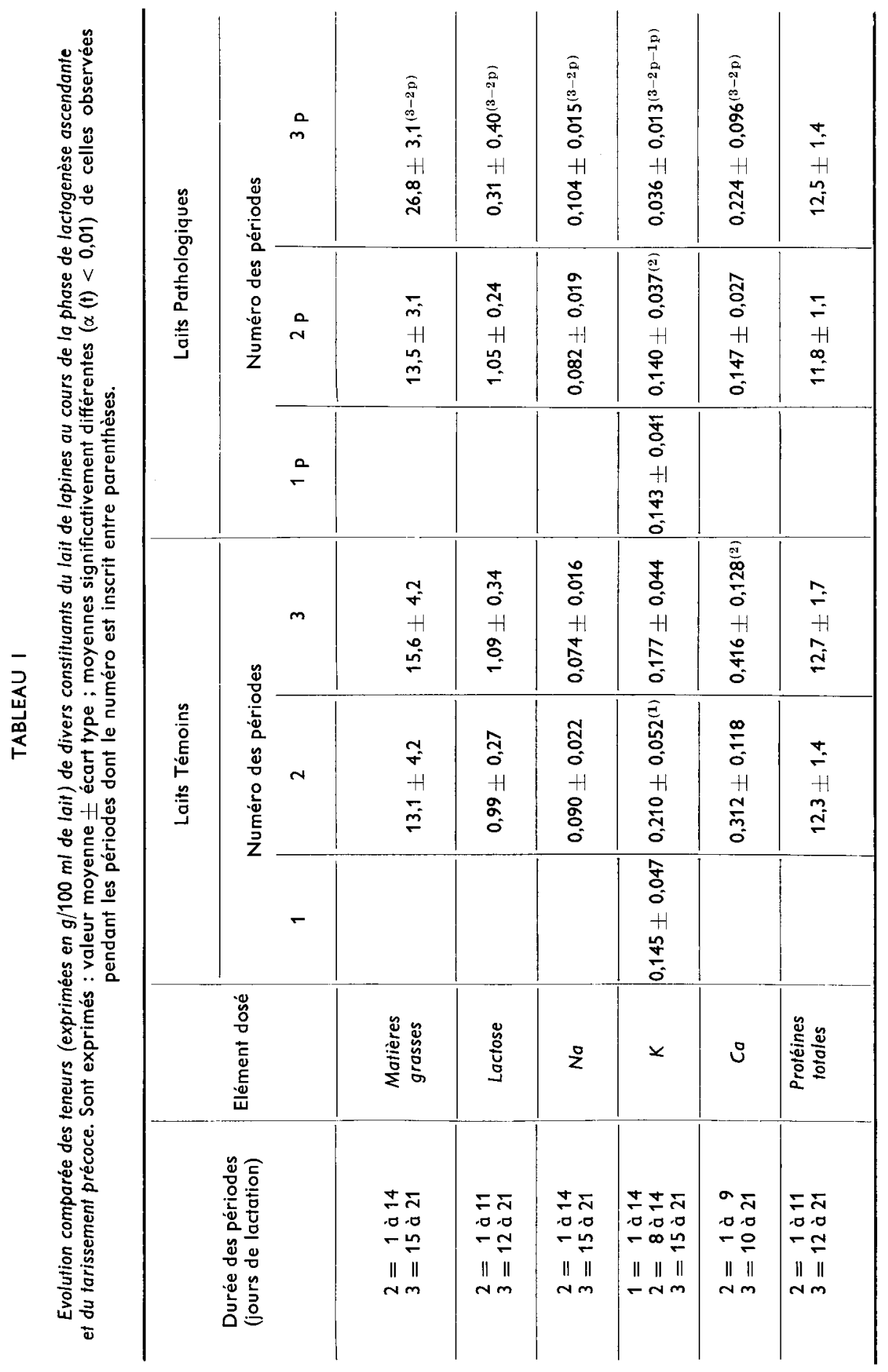


La teneur du lait en calcium augmente significativement pendant la seconde moitié de cette période. On note une hausse significative mais transitoire du taux de potassium au cours de la seconde semaine de lactation.

2) Les proportions de protéines, lactose, matières grasses, sodium et potassium des laits de farissement précoce (lot 2) sont semblables à celles observées dans les laits normaux (lot 1) pendant la première moitié de la période de lactation étudiée. Seul le taux de calcium est significativement plus faible.

3) Au-delà du $10^{\mathrm{e}}$ jour de lactation, on note pour le lot 2 :

a) une hausse significative des teneurs en matières grasses, sodium et calcium (cette dernière restant cependant significativement inférieure à celle observée dans les laits du lot 1);

b) une baisse significative des teneurs en lactose et potassium ;

c) que le taux de protéines reste stable et semblable à celui observé dans le lot 1. La composition grossière de ces protéines reste elle-aussi très constante. Dans les deux lots et pour l'ensemble de la période étudiée, elles sonf composées de $58,6 \pm 1$ p. 100 de caséine.

B. - L'analyse fractionnée (sur couche mince) des matières grasses du lait de la lapine montre que les triglycérides en sont invariablement l'élément majeur. Les autres fractions étant : des acides gras libres, des phospholipides et du cholestérol. Les variations du taux des matières grasses sont liées à celles des triglycérides. L'analyse de leur composition permet de noter que :

\section{TABLEAU ॥}

Evolution comparée de la composition (exprimée en p. cent de l'ensemble des triglycérides ou des acides gras) des friglycérides du lait de lapines témoins ou en cours de tarissement. Sont exprimés : valeur moyenne \pm écart type ; moyennes significativement différentes $(\alpha(t)<0,01)$ de celles observées pendant la période dont le numéro est inscrit entre parenthèses.

\begin{tabular}{|c|c|c|c|c|}
\hline \multirow[b]{2}{*}{ Numéro des Périodes } & \multicolumn{2}{|c|}{ Laits Témoins } & \multicolumn{2}{|c|}{ Laits Pathologiques } \\
\hline & 1 & 2 & $1 \mathrm{p}$ & $2 p$ \\
\hline $\begin{array}{l}\text { Durée des Périodes } \\
\text { (Jours de lactation) }\end{array}$ & 1 à 10 & 11 à 21 & 1 à 10 & 11 à 19 \\
\hline $\begin{array}{l}\text { Triglycérides « courts » } \\
(C \leqslant 36) \ldots \ldots \ldots \ldots\end{array}$ & $23,7 \pm 5,3$ & $42,6 \pm 10,3^{(1)}$ & $34,8 \pm 5,6^{(1)}$ & $35,5 \pm 9,6$ \\
\hline $\begin{array}{l}\text { Triglycérides « moyens }) \\
\quad(36<C<46) \ldots \ldots \ldots\end{array}$ & $36,4 \pm 3,8$ & $36,0 \pm 5,1$ & $42,6 \pm 1,5^{(1)}$ & $38,9 \pm 2,7^{(1 \mathrm{p})}$ \\
\hline $\begin{array}{l}\text { Triglycérides « longs » } \\
\quad(C \geqslant 46) \ldots \ldots \ldots . . .\end{array}$ & $39,9 \pm 7,3$ & $21,4 \pm 7,9^{(1)}$ & $22,6 \pm 5,7^{(1)}$ & $25,6 \pm 9,6$ \\
\hline $\begin{array}{l}\text { Acides gras « courts » } \\
(C \leqslant 10) \ldots \ldots \ldots . .\end{array}$ & $35,8 \pm 7,2$ & $40,6 \pm 6,8$ & $39,8 \pm 2,9$ & $38,8 \pm 7,6$ \\
\hline $\begin{array}{l}\text { Acides gras } « \text { moyens } » \\
\left(C_{12}-C_{14}\right) \ldots \ldots \ldots \ldots \ldots \ldots\end{array}$ & $4,5 \pm 0,7$ & $4,8 \pm 1,1$ & $4,2 \pm 0,2$ & $4,1 \pm 0,6$ \\
\hline $\begin{array}{l}\text { Acides gras } « \text { longs } » \\
\quad(C \geqslant 16) \ldots \ldots \ldots \ldots .\end{array}$ & $59,7 \pm 4,2$ & $54,6 \pm 3,3$ & $56,0 \pm 2,5$ & $57,1 \pm 6,6$ \\
\hline
\end{tabular}




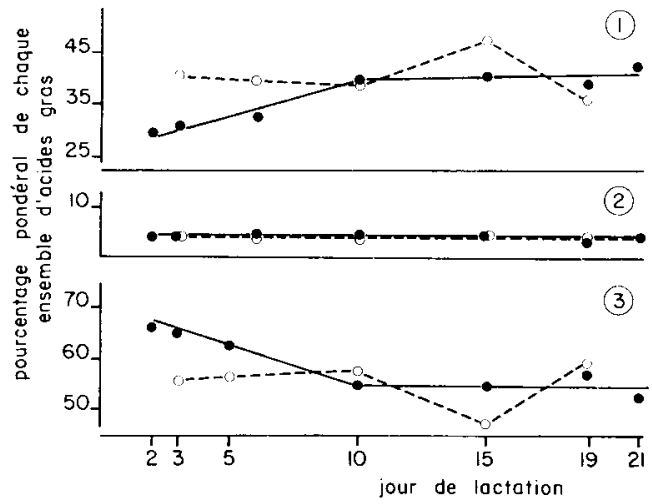

FIG. 1. - Evolutions comparées de la composition en acides gras des triglycérides du lait de lapines témoins (traits pleins) et de lapines souffrant de tarissement précoce (traits pointillés).

On a regroupé les acides gras dont la longueur de chaîne carbonée, $C$, est :

$1: C \leqslant 10 \quad 2: 10<C<16 \quad 3: C \geqslant 16$.

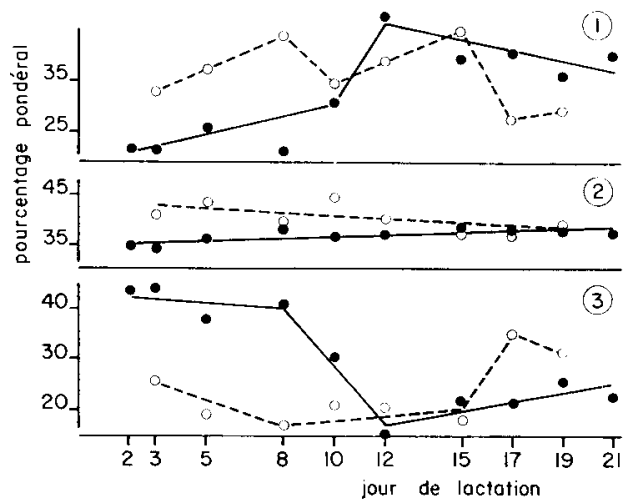

FIG. 2. - Evolutions comparées de la composition des groupes de triglycérides du lait de lapines témoins (traits pleins) et de lapines souffrant de torissement précoce (traits pointillés).

On a regroupé les triglycérides dont la somme des atomes de carbone des 3 acides gras, C, est :

$1: C \leqslant 36 \quad 2: 36<C<46 \quad 3: C \geqslant 46$.
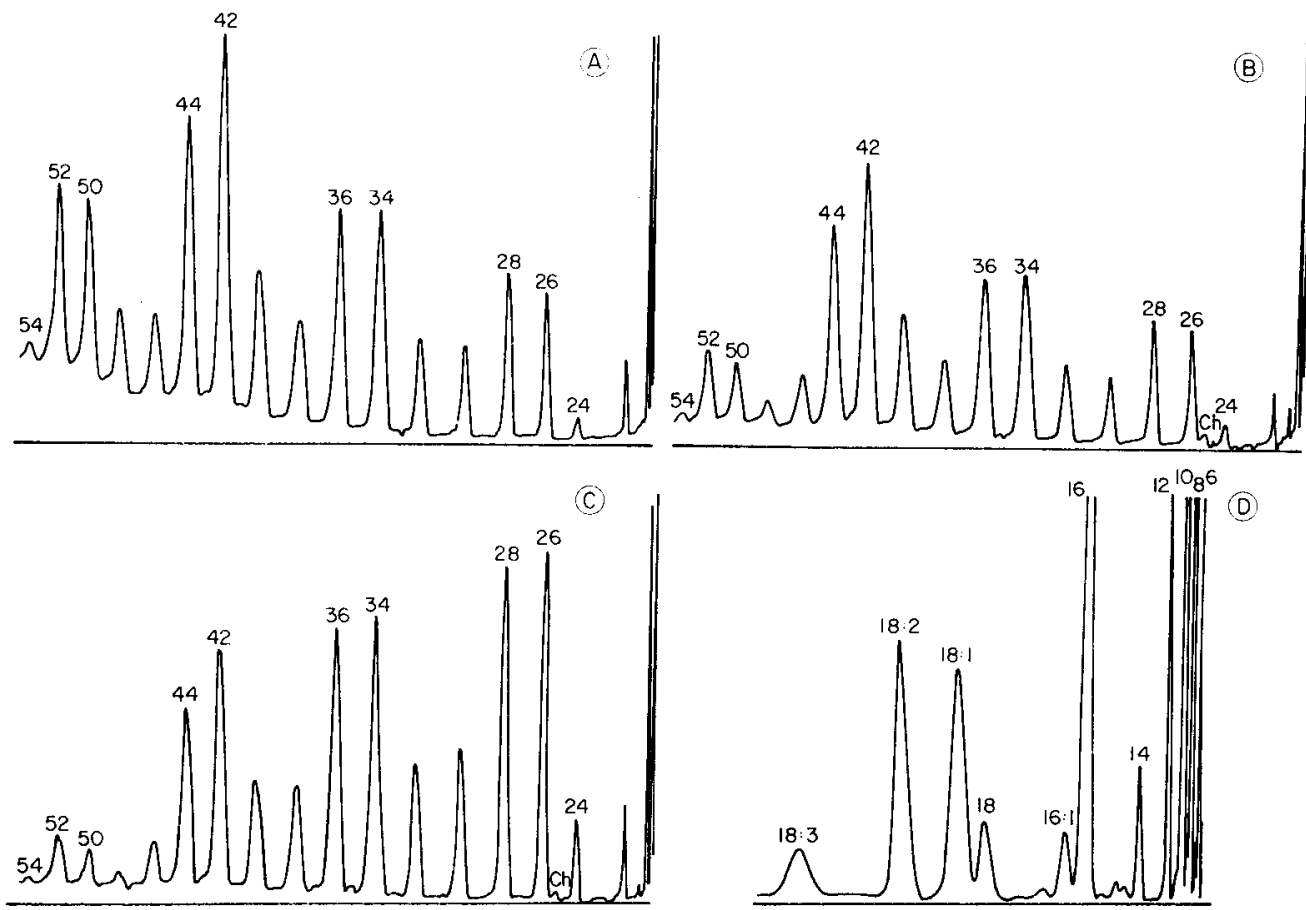

FIG. 3. - A-B-C. - Analyse des groupes de triglycérides de laits de lapine prélevés le troisième (A), le septième (B) et le seizième (C) jour de lactation. Chaque pic est identifié par le nombre total d'atomes de carbone des 3 acides gras constitutifs des divers triglycérides du groupe $(\mathrm{Ch}=$ cholestérol). D. - Analyse des acides gras des triglycérides d'un lait prélevé le onzième jour de lactation. Chaque acide est identifié par $x: y$, où $x$ représente le nombre d'atomes de carbone et $y$ le nombre de doubles liaisons dans la molécule. 
1) Dans les deux lots, les proportions des acides gras constitutifs des triglycérides sont semblables et n'évoluent pas. significativement. On observe pourtant une hausse progressive du taux des acides les plus courts ef surtout de l'acide décanoïque aux dépens de celui des acides les plus longs, pendant la première moitié de la phase de lactogenèse ascendante. Si la longueur de chaîne carbonée moyenne des acides gras est relativement stable dans les laits de tarissement : 13,5 en début de lactation et 13,9 en fin de lactation, elle diminue progressivement dans les laits normaux où sa valeur passe de 14,3 à 13,3. Les acides gras sont constitués de 35 à 40 p. 100 d'acides courts ou moyens $\left(C_{6}-C_{8}-C_{10}\right)$ et 54 à 60 p. 100 d'acides longs $\left(C_{16}-C_{18}\right)$. L'acide gras le plus court est l'acide caproīque $\left(C_{6}\right)$. On ne trouve que des traces d'acides gras à plus de 18 carbones. On relève destraces d'acides gras impairs $\left(C_{15}-C_{17}\right): \leqslant 0,1$ p. 100.

2) La répartition des acides gras dans les groupes de triglycérides varie significativement en fonction du stade de lactation et entre les deux lots (tabl. 2 et fig. 2). L'étude de l'évolution des proportions des divers groupes de triglycérides du lait nous a permis de distinguer trois ensembles qui évoluent de manières différentes:

- les triglycérides «longs » (dont le nombre d'atomes de carbone des 3 acides gras (C) est supérieur ou égal à 46) ;

- les triglycérides «moyens»: $36<\mathrm{C}<46$;

- les triglycérides « courts» $: C \leqslant 36$.

L'évolution de la répartition des acides gras entre les diverses molécules de triglycérides possibles est particulièrement nette si on compare ces répartitions avant et après le dixième jour de lactation (qui correspond au milieu de la période étudiée) et ceci dans les laits normaux. On note une inversion du rapport TG «longs»/TG « courts » qui passe de 1,86 en début de lactation à 0,46 après le $10^{e}$ jour. Pour les laits de tarissement, cette évolution est discrète et la valeur du rapport passe de 0,54 à 0,72 .

C. - La composition expérimentale des triglycérides comparée à celle calculée en supposant une répartition au hasard des acides gras dans les molécules de triglycérides permet de noter que (tabl. 3) :

1) l'on observe les mêmes différences dans les laits des deux lots ;

2) ces différences sont les suivantes :

a) absence de certains triglycérides possibles : $18-20-22$,

b) taux significativement supérieur de : $32-38-40$,

c) taux significativement inférieur de : $26-28$.

Le taux élevé du groupe de triglycérides 54 n'est significatif que dans les laits témoins alors que le taux réduit des groupes de triglycérides 42 el 44 ne l'est que dans les laits pathologiques ;

3) l'indice de spécificité global des triglycérides est semblable pour les laits des deux lots (łabl. 3) ; 
4) il varie peu en fonction du stade de lactation : 18,1 en début et 17,4 en fin de lactation pour les laits normaux ; 15,3 en début et 14,5 en fin de lactation pour les laits de tarissement. Ces valeurs moyennes ne sont pas différentes au seuil $\alpha(t)<0,10$.

TABLEAU III

Comparaison des compositions expérimentales $(E)$ ef colculées en supposant une répartition au hasard de leurs acides gras constifutifs (C) des triglycérides des laits témoins ef pathologiques. Valeurs moyennes de 18 analyses couvrant toute la période de lactation éfudiée.

* : moyennes significativement différentes $(\alpha(t)<0,01) \Sigma C$ - E : indice de spécificité calculé selon Watts ef al.

\begin{tabular}{|c|c|c|c|c|c|c|}
\hline \multirow{2}{*}{$\begin{array}{l}\text { Groupe de } \\
\text { Triglycérides }\end{array}$} & \multicolumn{3}{|c|}{ Laits Témoins } & \multicolumn{3}{|c|}{ Laits Pathologiques } \\
\hline & E & C & $C-E$ & $E$ & C & $\mathrm{C}-$ \\
\hline 18 & 0 & 0,05 & 0,05 & 0 & 0,07 & 0,07 \\
\hline 20 & 0 & 0,25 & 0,25 & 0 & 0,40 & 0,40 \\
\hline 22 & 0 & 0,75 & 0,75 & 0 & 1,00 & 1,00 \\
\hline 24 & 0,83 & 1,35 & 0,52 & 1,06 & 1,80 & 0,74 \\
\hline 26 & 4,40 & $1,83 *$ & & 5,10 & $2,14 *$ & \\
\hline 28 & 4,93 & $2,15 *$ & & 5,26 & $2,04 *$ & \\
\hline 30 & 2,82 & 2,97 & 0,15 & 2,98 & 3,30 & 0,32 \\
\hline 32 & 3,30 & $5,42 *$ & 2,12 & 3,82 & $6,60 *$ & 2,78 \\
\hline 34 & 8,48 & 8,20 & & 9,70 & 9,30 & \\
\hline 36 & 7,37 & 7,87 & 0,50 & 7,78 & 8,06 & 0,28 \\
\hline 38 & 3,45 & $5,63 *$ & 2,18 & 3,42 & $5,12 *$ & 1,70 \\
\hline 40 & 4,43 & $6,75 *$ & 2,32 & 5,20 & $6,96 *$ & 1,76 \\
\hline 42 & 14,35 & 12,80 & & 16,52 & $12,58 *$ & \\
\hline 44 & 14,87 & 14,35 & & 15,98 & $13,28 *$ & \\
\hline 46 & 6,43 & 8,33 & 1,90 & 6,20 & 7,10 & 0,90 \\
\hline 48 & 3,75 & 3,42 & & 2,74 & 2,18 & \\
\hline 50 & 8,12 & 4,97 & & 5,26 & 5,46 & 0,20 \\
\hline 52 & 9,87 & 8,87 & & 6,98 & 7,61 & 0,63 \\
\hline 54 & 2,60 & $5,98 *$ & 3,38 & 2,00 & 5,00 & 3,00 \\
\hline Indice de Spécificite & & & 14,12 & & & 13,78 \\
\hline
\end{tabular}

\section{IV. - Discussion et conclusion.}

L'étude de l'évolution de la qualité des matières grasses du lait de la lapine a été entreprise en écartant le plus possible les causes de variabilité dues à la race, la nourriture, la saison, l'abreuvement.

Les travaux de Lebas (1971), et la comparaison des données fournies par les auteurs ayant étudié la composition globale du lait des lapines en partant d'échantillons réduits ne permettent pas de penser que celle-ci dépende du stade de la traite à la différence de ce qu'on observe dans l'espèce humaine et chez la vache : Johansson (1952), la brebis : Labussière (1969), ef la truie : Salmon-Legagneur (1965), pour lesquelles l'intervalle entre les tétées est réduit. C'est pourquoi nous avons limité les prélèvements à $10 \mathrm{ml} /$ jour, car la traite est courte et le mamelon n'est pas traumatisé, ce qui évite de perturber le comportement maternel. 
L'étude de la composition chimique grossière des laits prélevés sur des lapines dont la lactation est normale, ne permet pas de déceler de différences essentielles avec les données de nos prédécesseurs, surtout au niveau des éléments minéraux. Les seules variations observées par ailleurs paraissent dépendre de la race. Nos lapins présentent un taux de matières grasses supérieur à celui décrit pour la race Californienne : Lebas (1971), égal à celui décrit pour la race New-Zealand : Coates (1964), Hall (1971) et inférieur à celui décrit pour la race Dutch : Hall. La teneur en protéines est semblable à celle des New-Zealand mais inférieure à celle des Californiennes. Pour le lactose, c'est l'inverse.

L'étude détaillée de la composition de leurs triglycérides permet de mettre en évidence des variations dans la qualité des matières grasses du lait, que l'analyse de leurs seuls acides gras constitutifs ne permet pas de déceler, tout au moins au cours des trois premières semaines de lactation. Hall (1971) avait mis en évidence une hausse de la teneur en acides gras moyens $\left(C_{8}\right.$ et $\left.C_{10}\right)$ aux dépens des trois principaux acides longs $\left(C_{16}-C_{18: 1}-C_{18: 2}\right)$ surtout en fin de lactation. Pendant les trois premières semaines, cette hausse est assez discrète. Elle n'est pas significative au seuil $\alpha=0,05$ d'après l'analyse statistique des données que nous avons obtenues.

Les proportions des divers acides gras composants les triglycérides sont proches de celles données par Hall, mais assez différentes de celles obtenues par Smith (1968), qui ont tous deux utilisé des lapines New-Zealand. L'écart peut être dû au mode de prélèvement du lait : Smith travaillant sur l'exsudat de glande mammaire fraîchement isolée. De plus, nous avons trouvé systématiquement une forte proportion d'acide caproïque $\left(C_{6}\right)$ dont la présence n'est signalée qu'à l'état de traces par Smith, Watts et Dils (1968).

L'évolution de la nature des différents triglycérides du lait est caractéristique. Elle n'a jamais été décrite. Si le profil général de cette composition est tout à fait semblable à celui donné par Smith, avec une dominance constante des triglycérides : $\mathrm{C}=26$ $-28-34-36-42-44$, nous notons par ailleurs une teneur élevée en : $C=50$ et 52 qui ne devient semblable à celle calculée par Smith qu'au-delà du dixième jour de lactation.

L'étude systématique de l'évolution des proportions des divers triglycérides nous a amené à en distinguer trois types dont la variation du taux est significative et rend compte de l'évolution progressive de la qualité des matières grasses du lait. Ainsi le pourcentage de triglycérides « courts» $(C<36)$ augmente considérablement au-delà du dixième jour de la lactation à l'inverse de celui des triglycérides « longs" $(C>46)$. Cette évolution n'existe pas dans les laits de tarissement.

Ce stade de l'évolution de la lactation, situé au-delà du dixième jour après la mise bas apparaît essentiel. Il est d'ailleurs mis en évidence par l'ensemble des résultats que nous avons obtenus. Une évolution défavorable est simultanée de la hausse du taux de $\mathrm{Na}$ et de matières grasses et de la chute du taux de $\mathrm{K}$ et de lactose. La hausse du taux des matières grasses est accompagrée par le maintien d'une faible proportion de triglycérides « courts».

La variation de ces paramètres est conforme à celle décrite dans les laits de fin de lactation d'autres espèces et accompagne la chute de la production lactée. Mais l'évolution de deux autres indices précède catte chute. 
Ainsi les taux de calcium et de triglycérides courts sont significativement plus faibles dès ce début de la lactation, alors que la production lactée est convenable en quantité et la survie des lapereaux assurée. La mesure de ces deux indices permettrait donc de prévoir le tarissement précoce.

Ces laits de tarissement ont une composition en acides gras peu différente de celle des laits témoins. Leur teneur en acides gras courts est un peu plus élevée pendant les dix premiers jours de la lactation. Le taux des triglycérides courts est significativement plus élevé.

Ceci peut être dû au fait que les acides gras courts sont incorporés de manière spécifique dans des triglycérides courts par la glande mammaire fabriquant des laits pathologiques. Mais nous n'avons pas relevé de différence dans les indices de spécificité globaux ou particuliers des triglycérides des deux lots de laits. Il ne semble pas que le processus d'estérification des acides gras varie avec le stade de lactation. Ceci est confirmé par le fait que l'indice de spécificité global des triglycérides reste stable pendant la lactogenèse ascendante pour les lapines témoins ou le tarissement précoce pour les autres.

Par ailleurs, les différences que nous avons relevées entre les compositions expérimentales ou calculées en supposant une répartition au hasard des acides gras constifutifs de ces triglycérides permetient d'envisager deux éventualités :

- les acides gras ne sont pas estérifiés au hasard,

- ou bien, ils sont estérifiés au hasard mais au sein d'ensembles distincts et de compositions différentes. On sait en effet que les acides gras disponibles pour la synthèse des triglycérides du laił ont deux origines : les plus courts sont synthétisés par la glande mammaire à partir de l'acétate, alors que les plus longs proviennent des acides gras libres plasmatiques et des acides gras des triglycérides des chylomicrons et des lipoprotéines (L.D.L.).

En l'état actuel de nos travaux, il n'est pas possible de dire si le taux élevé d'acides gras et de triglycérides courts dans les laits pathologiques du début de lactation est dô à une synthèse accrue d'acides gras courts ou à un défaut de captation des acides gras longs plasmatiques.

Accepté en septembre 1976.

\section{Références}

APPEL K. R., BUSSE H., SCHULZ K. D., WILK W., 1971. Beitrag zur Handaufsucht von gnotobiotischen und S. P. F. Kaninchen. Z. Vesuchstierke, 135, 282-290.

CAREY E. M., DILS R., 1973. Fatty acid biosynthesis X : Specificity for chain termination of fatty acid biosynthesis in cell free extracts of lactating rabbit mammary gland. Biochim. Biophys. Acta, 3C6, 156-167.

COATES M. E., GREGORY M. E., THOMPSON S. Y., 1964. The composition of rabbit's milk. Brit. J. Nutr., 18, 583-586.

COWIE A. T., 1969. Variations in the yield and composition of the milk during lactation in the rabbit and the galactopoietic effect of prolactin. J. Endocr., 44, 437-450.

DELSAL J. L., 1944. Nouveau procédé d'extraction des lipides du sérum par le méthylal. Application aux microdosages du cholestérol total, des phosphoaminolipides et des protéines. Bull. Soc. Chim. Biol.. 26, 99-105. 
DENAMUR R., 1961. Etude de la glande mammaire de la lapine pendant la gestation et la lactation. Ann. Endocr., 22, 768-776.

DILS R., FORSYTH I., STRONG C. R., 1972. Hormonal control of the synthesis of milk fatty acids by rabbit mammary gland. J. Physiol., 222, 94p-95p.

DORCHE J., MATHIAN P., AVANTURIER G., 1951. Sucres réducteurs : méthode générale de dosage. Lyon Pharmaceutique, 5, 139.

GUEGUEN L., ROMBAUTS P., 1961. Dosage du sodium, du potassium, du calcium et du magnésium par spectrophotométrie de flamme dans les aliments, le lait et les excréta. Ann. Biol. anim. Bicch. Biophys., 1, 80-97.

HALL A. J., 1971. Fatty acid composition of rabbit (Orytogalus cuniculus) milk fat throughout lactation. Inf. J. Biochem., 2, 414-418.

JOHANSSON I., KORKMAN N., NELSON N. J., 1952. Studies on udder evacuation in dairy cows. I : the rise in fat percentage during milking. Acta Agric. Scand., 2, 43-81.

KUKSIS A., MC CARTHY M. J., BEVERIDGE J. M. R., 1963. Quantitative gas liquid chromatographic analysis of butterfat triglycerides. J. Am. Oil Chem. Soc., 40, 530-535.

LABUSSIERE J., 1969. Importance, composition et signification des différentes fractions de lait obtenues successivement au cours de la traite mécanique des brebis. Ann. Zootech., 18, 185-196.

LEBAS F., 1971. Composition chimique du lait de lapine, évolution au cours de la traite et en fonction du stade de lactation. Ann. Zoofech., 20, 185-191.

LEBAS F., BESANÇON P., ABOUYOUB A., 1971. Composition minérale du lait de lapine. Variations en fonction du stade de lactation. Ann. Zoofech., 20, 487-495.

SALMON-LEGAGNEUR E., 1965. Quelques aspects des relations nutritionnelles entre la gestation ef la lactation chez la truie. Ann. Zootech., 14 (h. s.), 143 pp.

SMITH S., WATTS R., DILS R., 1968. Quantitative gas liquid chromatographic analysis of rodent milk triglycerides. J. Lip. Res., 9, 52-57.

WATTS R., DILS R., WEHR H. Analysis of human serum triglycerides by high temperature gas chromatography. J. Chromatog., 66, 239-247. 